SUBJECT AREAS:

MOTOR CONTROL

DYNAMICAL SYSTEMS

Received

22 April 2014

Accepted

3 July 2014

Published

1 August 2014

Correspondence and requests for materials should be addressed to

D.McG. (denise. mcgrath@ucd.ie)

\section{The influence of auditory-motor coupling on fractal dynamics in human gait}

\author{
Nathaniel Hunt ${ }^{1,2}$, Denise McGrath ${ }^{3}$ \& Nicholas Stergiou ${ }^{1}$
}

\begin{abstract}
'Biomechanics Research Building, University of Nebraska at Omaha, 6160 University Drive, Omaha, NE 68182-0860, ${ }^{2}$ Department of Integrative Biology, University of California, Berkeley, 3040 Valley Life Sciences Building \#3 140, Berkeley, CA 94720-3140, ${ }^{3}$ School of Public Health, Physiotherapy and Population Science, University College Dublin, Woodview House, Belfield, Dublin 4, Ireland.
\end{abstract}

Humans exhibit an innate ability to synchronize their movements to music. The field of gait rehabilitation has sought to capitalize on this phenomenon by invoking patients to walk in time to rhythmic auditory cues with a view to improving pathological gait. However, the temporal structure of the auditory cue, and hence the temporal structure of the target behavior has not been sufficiently explored. This study reveals the plasticity of auditory-motor coupling in human walking in relation to 'complex' auditory cues. The authors demonstrate that auditory-motor coupling can be driven by different coloured auditory noise signals (e.g. white, brown), shifting the fractal temporal structure of gait dynamics towards the statistical properties of the signals used. This adaptive capability observed in whole-body movement, could potentially be harnessed for targeted neuromuscular rehabilitation in patient groups, depending on the specific treatment goal.

revious research investigating sensorimotor synchronization suggests that when rhythms are presented in different modalities, there appears to be an auditory advantage over other sensory pathways. Sejdic et al. ${ }^{1}$ showed that, compared to visual and haptic rhythms, auditory rhythms have the strongest influence on a person's gait. An evolutionary reason for this advantage has been presented, suggesting that bipedal gait and the associated sounds of locomotion influenced the evolution of human auditory-motor rhythmic abilities ${ }^{2}$. Traditional finger-tapping paradigms have refined our understanding of the neural circuitry that underpins auditory-motor coupling, however the mechanisms underlying this phenomenon in whole-body, functional movements are not well understood. This is due to the practical limitations associated with brain imaging and measurement techniques currently available for upright, whole body movements. However, despite these practical limitations that render investigations into neural circuitry somewhat elusive for whole body movement, there is still a pressing need to capitalize on the potential that auditory-motor coupling offers the field of neuromuscular rehabilitation. The authors describe a novel auditory-motor entrainment paradigm that could potentially be implemented for the rehabilitation of gait, in cases of brain injury and neurodegenerative disease.

Synchronization of walking to rhythmic auditory cues has previously been investigated as a potential training tool to support functional independence in individuals with pathological gait ${ }^{3-5}$. Parkinson's disease and stroke, in particular, have been the main focus of auditory cueing investigations, with evidence to suggest that walking to a fixed tempo metronome may temporarily improve gait velocity, stride length, cadence and symmetry ${ }^{6,7}$. However, these studies have not taken the temporal dynamics of gait parameters into account, i.e. the sequencing of gait parameters over time, which reflects the adaptive state of the neuromuscular system. Well-established motor learning principles suggest that variability in practice and feedback is required for better learning outcomes ${ }^{8}$, however this is largely absent from the field of auditory cueing for gait rehabilitation, which has focused predominantly on fixed tempo metronomes as the auditory cue.

Gait variability can be defined as the natural stride-to-stride fluctuations that are inherent in normal, healthy gait $^{9}$. These stride-to-stride fluctuations are thought to arise from interconnected, nonlinear processes that enable us to expand and maintain a large repertoire of movement strategies, allowing robust gait in dynamic, unpredictable environments. Fractal patterns observed in biological signals such as heart rate ${ }^{10}$, respiration ${ }^{11}$ and walking strides ${ }^{12}$ measured over time, indicate that the time intervals between events are not equal, nor are they independent. Rather, there is a relationship between these intervals that extends far forward and backward in time; in other words, exhibiting long-range correlations in the time series, or fractal fluctuations. The presence of these fractal processes in biological systems is theoretically referred to as "complexity"13, which describes an underlying order or pattern that is contained within a complex, variable system; a system that is capable of sudden 


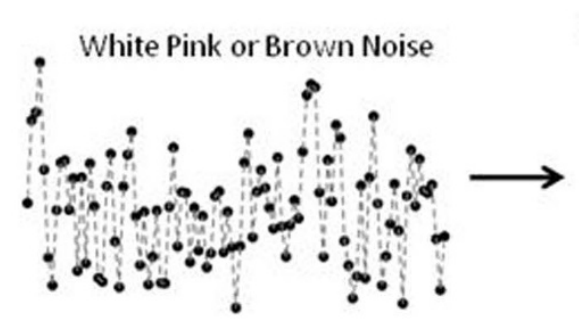

Individualized Rhythmic Auditory Stimulus

uliellimy

Inter-Stride Intervals

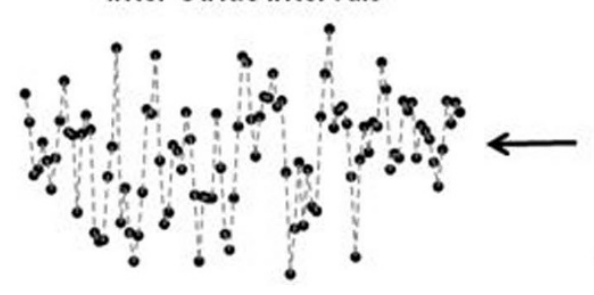

Footswitch Signal

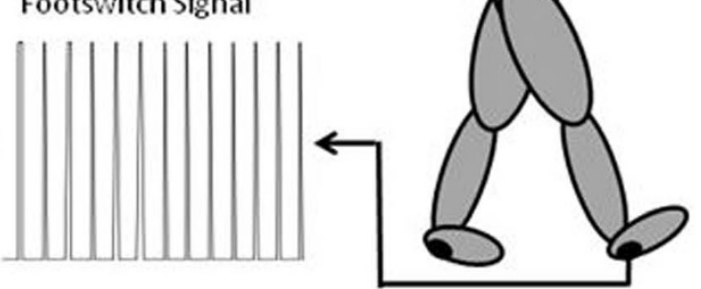

Figure 1 | Signal Flow of Experiment.

and marked change. The multi-scale fractal structure of the relationships between gait events is therefore thought to be ordered and stable, yet variable and flexible.

Complexity is recognized as an inherent attribute of healthy biological systems, whereas the loss of complexity with aging and disease is thought to reduce the adaptive capabilities of the individual ${ }^{12,14-19}$. A loss of complexity can refer to either an overly constrained, periodic system, or an overly random, incoherent system ${ }^{20}$. Quantitative measures of the temporal structure of human gait variability - such as statistical persistence and the amount of information present within the motor time series - provides us with a window into the organization of the neuromuscular system ${ }^{21}$. Increasingly, abnormal gait patterns can be characterized by the altered temporal structure of gait variability, and have been associated with a variety of disorders ranging from joint and skeletal problems ${ }^{22}$ (lowest-level gait disturbances) to Huntington's disease ${ }^{23}$, Parkinson's disease ${ }^{24}$, higher level gait disorders ${ }^{25}$ and falls ${ }^{26-29}$.

It follows, then, that if a healthy neuromuscular system exhibits an optimal state of movement variability ${ }^{9}$, gait rehabilitation practices should also embody this conceptual module into the auditory-motor entrainment rehabilitation paradigm. Indeed, it has been empirically demonstrated that our nervous systems can actively exploit motor variability to facilitate motor learning, with increased rate of learning in individuals who exhibit higher levels of behavioural variability ${ }^{30}$. However, with the exception of a small number of recent studies that have explored the use of complex auditory cues during walking ${ }^{31-33}$, most rhythmic auditory cueing gait research has focused on a fixed tempo metronome. It is now quite clear from the literature that walking in time to a fixed tempo metronome alters the natural dynamics of human gait, shifting the structure away from the persistent fractal structure towards more anti-correlated behaviour $^{1,32-37}$. Advances in this field require a multidisciplinary approach to devise an auditory cueing paradigm that could potentially restore the complex dynamics that are known to exist in healthy gait.

A review of evidence in finger tapping suggests that tapping to rhythmic sequences of differing temporal structures recruits neural circuitry differently ${ }^{38}$. Recent studies by the authors ${ }^{33}$ and Marmelat et $\mathrm{a}^{32}$ have investigated to what extent human gait can be controlled using complex auditory signals during treadmill walking. The results of these studies suggest that auditory signals can potentially be optimized for gait retraining using a complexity based approach. Here we shift our focus to overground walking thus removing the potentially confounding constraint of the treadmill ${ }^{39,40}$. This study sought to systematically manipulate the nonlinear statistical properties of auditory stimuli that were embedded into familiar music, in terms of predictability and statistical persistence of inter-beat intervals. We hypothesized that participants would exhibit differences in gait dynamics that depended on which auditory signal they listened to while walking. The premise of this study was as follows: if the auditory-motor entrainment phenomenon proved to be sufficiently adaptive in terms of mapping the global dynamics of a nonlinear auditory system, this opens up an exciting new avenue for the reorganization of the neuromuscular system in injury or disease.

\section{Methods}

Participants. Ten healthy participants (4F, 6M; $28.1 \pm 5.3 \mathrm{yrs} ; 175 \pm 11.4 \mathrm{~cm} ; 72.3$ $\pm 16.2 \mathrm{~kg}$ ) were recruited for this study. The study was approved by our University's Medical Center Institutional Review Board, and the study was carried out in accordance with the approved guidelines. Each subject provided informed consent prior to participation.

Experimental design. The goal of this study was to manipulate a person's gait dynamics using an individualized fractal rhythmic auditory stimulus (IFRAS). We embedded three different levels of noise into a well-known melody by manipulating the inter-beat interval (Fur Elise by Beethoven) - white noise (representing fully random, unpredictable processes with no correlations in time), brown noise (representing processes that are stable but unable to flexibly modify their activity when rapid changes are required) and pink noise (or 1/f noise, representing fractal processes that are both stable and flexible, as discussed above in relation to 'complexity'). We asked the subjects to walk in time to the music, and analysed the temporal structure of their resultant gait dynamics to determine the level of auditorymotor entrainment with each IFRAS in terms of global statistical structure. A schematic of the experimental design is presented in Figure 1.

Experimental protocol. Walking trials were conducted indoors around an $1 / 8^{\text {th }}$ mile oval track, clear of other users. Subjects wore a portable Stride Analyzer (Stride Analyzer, B.L. Engineering, Santa Ana, CA) to collect heel strike times. Subjects wore AKG K-55 over the ear headphones (AKG Acoustics, Vienna, Austria). Headphones received the music signal played from an Olympus WS-600S digital voice recorder (Olympus Corporation, Shinjuku, Tokyo, Japan). An initial data collection of overground walking was conducted with no auditory stimulus to determine the participants' preferred cadence and the standard deviation of their step time. These two parameters were used to create the IFRAS for the three types of music so that the cadence and the distribution of the music matched the individual's walking pattern. Each participant then performed three subsequent walking trials in a randomized order with 10 minutes between each condition, listening to the white noise music, the pink noise music and brown noise music (supplementary material 1). Participants walked on the indoor track for approximately 612 strides for each trial (this is accomplished by using a trial time that is the step cadence of the individual subject multiplied by 1224). At the beginning of each trial the researcher stated the following instructions:

"This may be very difficult but I would like you to try your best to concentrate on the task throughout the entire walking trial. Listen carefully to the music and also pay close attention to when your heel hits the ground. I would like you to walk normally but think about the note that is coming next and imagine that you are playing the notes with your 

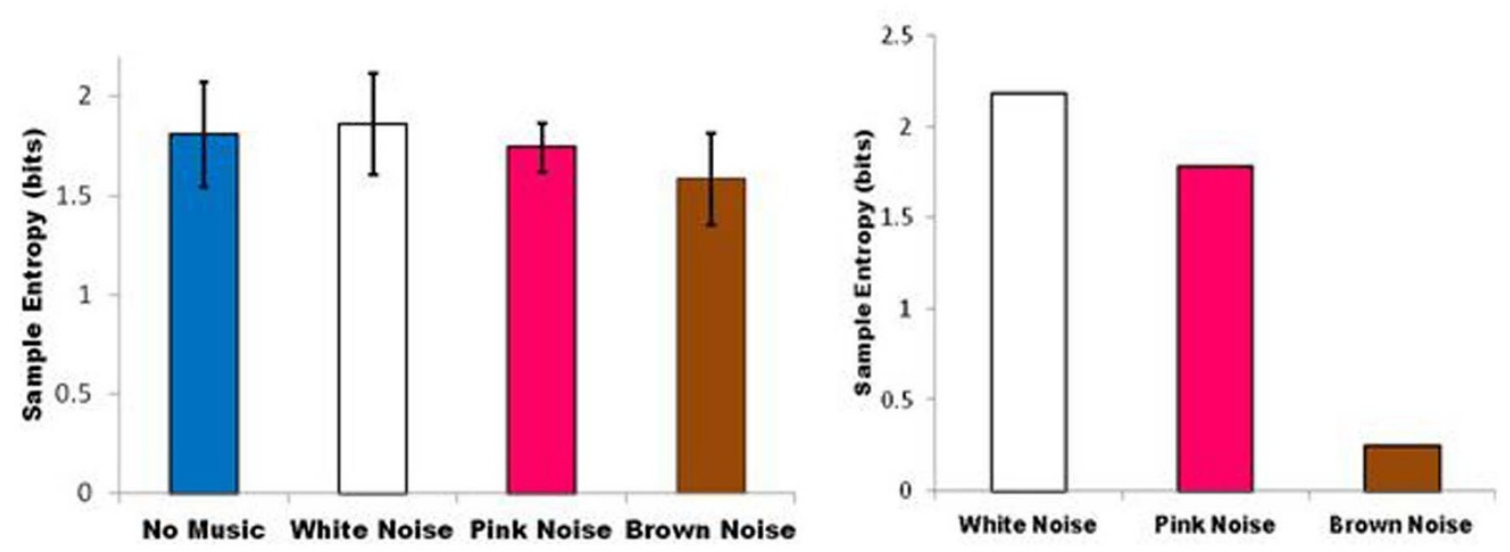

Figure 2 Sample entropy analysis of the auditory signal is shown on the right for illustration purposes. Sample entropy analysis of the stride time series across the four different gait conditions for all subjects is shown on the left. Error bars are standard deviations.

heel strikes. Every time around the track, I will hold up a sign to remind you to concentrate on playing the notes with your heel-strikes. When the trial is over I will tell you."

The instructions were given as such to facilitate synchrony between the subject and the music by (1) asking them to anticipate the upcoming note of the song, and (2) emphasizing the link between the note timing and the heel strike timing.

Data collection. Individualized fractal rhythmic auditory stimulus. In music, the amount of time between two successive notes is the inter-beat interval. In gait, an analogous quantity to the inter-beat interval is the step time (the amount of time between successive heel-strikes of the contralateral feet). The IFRAS was generated by taking a white noise, pink noise, or brown noise time series and embedding that variable into the inter-beat interval of a piece of music. The first section of the song Fur Elise by Beethoven was chosen both because it is widely familiar, and because it can be played with identical inter-beat intervals between each note without gross modification to the musical composition. Hearing familiar music conjures anticipatory auditory imagery. This auditory imagery increases the accuracy of auditory-motor synchronization when compared to a metronome $\mathrm{e}^{41}$. For the white noise, a random time series was generated by the MATLAB rand() function. The brown noise theoretical time series was created by integrating the white noise time series. The pink noise time series was generated by filtering brown noise. A weighted sum of first order filters was used so that the power drops off at $-10 \mathrm{~dB}$ per decade. It is accurate to within $+/-0.05 \mathrm{~dB}$. All the music was generated using Musical Instrument Digital Interface files so that the onset of each note can be designed precisely and the inter-beat interval dynamics can be controlled. For each participant, the amplitude of the perturbation was individualized. The inter-beat interval was stretched or compressed depending upon the dynamics of the corresponding coloured noise time series. The amplitude was determined such that the standard deviation of the inter-beat intervals matched the standard deviation of the step times of the subject. Also, the tempo of the music was modified to match the preferred cadence of each subject.

Gait parameters. Stride times were measured using a Stride Analyzer. This device encodes on/off timing for multiple footswitches embedded in shoe insoles. It records this timing information in a binary format. Custom MATLAB code was written to decode the binary files and give the on/off timing of the heel switch.

Data analysis. All subjects walked at least 588 strides per trial. The first 50 strides of each trial were discarded prior to analysis to mitigate any transient effects related to the initiation of walking with the IFRAS auditory stimulus. The subsequent 512 strides were analyzed, in keeping with recommendations for short time series ${ }^{42}$. All subjects and all trials were included in the analysis. Data were inspected for differences between right and left legs. For all trials we collected the following parameters:

Sample entropy of the stride-interval time series was calculated for each IFRAS condition ${ }^{43}$. Entropy algorithms applied to stride times provide a quantification of the uncertainty or the unpredictability of the stride-to-stride fluctuations over continuous gait cycles. The fractal scaling exponent, $\alpha$, of the stride-interval time series was calculated using the Detrended Fluctuation Analysis (DFA) method (box sizes range: 2-32). This measure allowed comparisons of the resultant gait dynamics between no music, white noise music, pink noise music and brown noise music, and gives partial evidence of long-term dependencies within the observed stride-to-stride fluctuations. Entropy and the fractal scaling exponent are nonlinear measures that have identified differences in biological signals between healthy and pathological populations ${ }^{10,44,45}$.
Deterioration in these measures signify reduced connectivity in motor control processes and appear to be important in diagnosing and characterizing pathological gait $^{23,26,46,47}$.

Statistical analysis. Mean values for the dependent variables $-\alpha$, fractal scaling exponent and sample entropy of stride interval time series - were compared across auditory stimulus and no music conditions with a one-way repeated measures ANOVA. Mauchly's test was implemented to test for sphericity. The alpha level was set at 0.05 . Post-hoc pairwise comparisons were performed, with an adjusted alpha level for multiple comparisons. Trend analysis was performed across conditions when ordered according to the statistical properties of the auditory stimuli e.g. increasing scaling exponent from white noise, followed by pink noise, followed by brown noise. Statistical analyses were performed in SPSS software (SPSS Inc., Chicago, IL). Omega squared $\left(\omega^{2}\right)$ for a repeated measures design was used as an unbiased measure of effect size suitable for small samples; effect size, $r$, was used for pairwise comparisons.

\section{Results}

No differences were observed between the right and left legs. All results are reported in relation to the right leg. Mean and standard deviation of stride times across all subjects when walking without an auditory stimulus was 1.07 (0.11) seconds and mean standard deviation across all subjects was 0.02 seconds. Mauchly's test indicated that the assumption of sphericity had not been violated for either outcome variable (sample entropy: $\mathrm{x}^{2}(5)=7.376, \mathrm{p}=0.197$; DFA: $\left.\mathrm{x}^{2}(5)=2.515, \mathrm{p}=0.776\right)$. There was a significant effect of auditory stimulus on the sample entropy of the stride interval time series $[\mathrm{F}(1,9)=4.465 \mathrm{p}=0.011]$, with an effect size $\omega^{2}=0.15$. A significant linear trend between auditory signal conditions was observed ( $\mathrm{p}=$ 0.026 ), indicating that the brown noise walking condition yielded lower sample entropy of stride times than the pink noise condition, followed by the white noise walking condition, mirroring the sample entropy trends inherent in the actual auditory signals themselves i.e. brown noise lower than pink noise, lower than white noise (Figure 2). Effect size calculations between conditions suggest that the brown noise condition led to greatest changes in sample entropy of stride times, with large effect sizes $\left(\mathrm{r}>0.5^{48}\right)$ observed across all brown noise comparisons (Table 1). Similarly, there was a significant effect of auditory stimulus on the fractal scaling exponent of the stride interval time series $[\mathrm{F}(1,9)=3.179, \mathrm{p}=0.04]$, with an effect size $\omega^{2}=0.12$. A significant linear trend between conditions was observed $(\mathrm{p}=0.004)$, indicating that the pink noise and brown noise walking conditions yielded higher fractal scaling exponents - or higher statistical persistence - than the white noise condition, mirroring the fractal scaling trends in the actual auditory signals themselves i.e. brown noise higher than pink noise, higher than white noise (Figure 3). Effect size calculations between conditions suggest 
Table $1 \mid$ p-values and effect sizes for pairwise comparisons

\begin{tabular}{lllllll} 
& \multicolumn{2}{c}{ Sample Entropy } & & \multicolumn{2}{c}{ Scaling Exponent } \\
\cline { 2 - 3 } Conditions & $p$-value & Effect Size $r$ & & $p$-value & Effect Size $r$ \\
\hline No Music v White Noise & 0.52 & 0.22 & & 0.015 & 0.71 \\
No Music v Pink Noise & 0.52 & 0.22 & & 0.557 & 0.2 \\
No Music v Brown Noise & 0.017 & 0.7 & & 0.974 & 0.01 \\
White Noise v Pink Noise & 0.1 & 0.52 & & 0.048 & 0.61 \\
White Noise v Brown & $0.004 *$ & 0.79 & & 0.023 & 0.67 \\
Noise & & & & & \\
Pink Noise v Brown Noise & 0.048 & 0.61 & 0.51 & 0.22 \\
\hline *denotes significance at $p<0.008$ (adjusted critical value for multiple comparison).
\end{tabular}

that the white noise condition led to greatest changes in the scaling exponent of stride times, with large effect sizes observed across all white noise comparisons ( $r>0.5$ ) (Table 1 ). In summary, an adaptation occurred in gait dynamics as a result of listening to the auditory stimuli. $12-15 \%$ of variance in the stride time dynamics is attributable to the structure of the stimulus. While the auditory stimulus modified the fluctuation pattern of gait, these young, healthy individuals broadly tended to produce pink noise in their stride-to-stride fluctuations across all conditions (i.e. scaling exponents between 0.75 (white noise condition) and 0.94 (brown noise condition)). However, the modifications occurred in a predictable direction, depending on the global statistical properties of the auditory stimulus. The brown and white noise music stimuli appeared to elicit the greatest adaptation.

\section{Discussion}

This study systematically altered the statistical structure of musical auditory cues during overground walking, and quantified the statistical structure of the resultant motor time series, both in terms of statistical persistence and sample entropy. Our findings demonstrate plasticity of the auditory-motor phenomenon during whole-body, upright movement, as the trend across the structure of fluctuations in the IFRAS is mirrored in the trend across the structure of fluctuations in the gait time series. Our findings add to the existing body of literature that demonstrates the fractal nature of stride interval time series produced by healthy, young adults, and additionally illustrate that this fractal structure is rather robust to auditory perturbation in overground walking. While the white and brown noise music conditions engendered an adaptation in gait dynamics towards the auditory cue's statistical structure, the absolute values of the scaling exponent and sample entropy did not stray very far from what might be considered the "normal" range for this population (e.g. scaling exponent between 0.7 and 1).

A recent study by Marmelat and colleagues ${ }^{32}$ investigated stride interval time series when listening to auditory stimuli with differing fractal structures in 12 young, healthy adults. These participants appear to have exhibited different levels of adaptation to the nonisochronous auditory stimuli, compared to the participants in the present study. For example, an auditory stimulus with a scaling exponent of 0.5 - analogous to our white noise music condition produced a stride interval time series of scaling exponent $0.44 \mathrm{com}-$ pared to 0.75 in our study, and an auditory stimulus with a scaling exponent of 0.9 - analogous to our pink noise music condition produced a stride interval time series of scaling exponent 0.64 compared to 0.89 in our study. The reasons for these differences are not immediately clear although the study protocols were quite different. The current study focused on overground walking with musical auditory stimuli, which, one might intuitively expect, would lead to stronger entrainment between signals compared to Marmelat et al.'s treadmill based protocol and non-musical stimulus, but this was not borne out in the results. Nonetheless, our study did result in predictable changes in statistical properties in terms of the direction of the changes in outcome measures, as opposed to their absolute values. This finding lends itself to interpretation within the 'strong anticipation' framework ${ }^{32,49-52}$, i.e. the master-slave ana$\operatorname{logy}^{52}$ : a strongly anticipatory system is one where "slave" (e.g., organism) is not adapted to the states of the "master" (e.g., environment), but rather is adapted to its statistical structure. However, given that the stride times and auditory signals were not precisely synchronized in our study, we cannot examine this interpretation appropriately.

In the interest of enquiry, we will discuss a number of future research questions evoked by the findings of this study, and previous work in this area. Our results showed differences in the way subjects responded to the IFRAS. One possible explanation for this variability within groups is previous musical training ${ }^{53}$. Within-group variability could also result from physical or cognitive impairments. Our subjects were healthy individuals, so the former explanation is more likely in this case. However, future research should investigate the ability to entrain to auditory cues with varying fractal fluctuations in specific patient populations with physical and cognitive impairments. This would lead to more targeted and successful interventions, with particular auditory signal structures potentially being more appropriate for particular deficits.

Previous work conducted by the authors ${ }^{33}$ studied a group of older adults, along with a group of younger adults to investigate the idea of
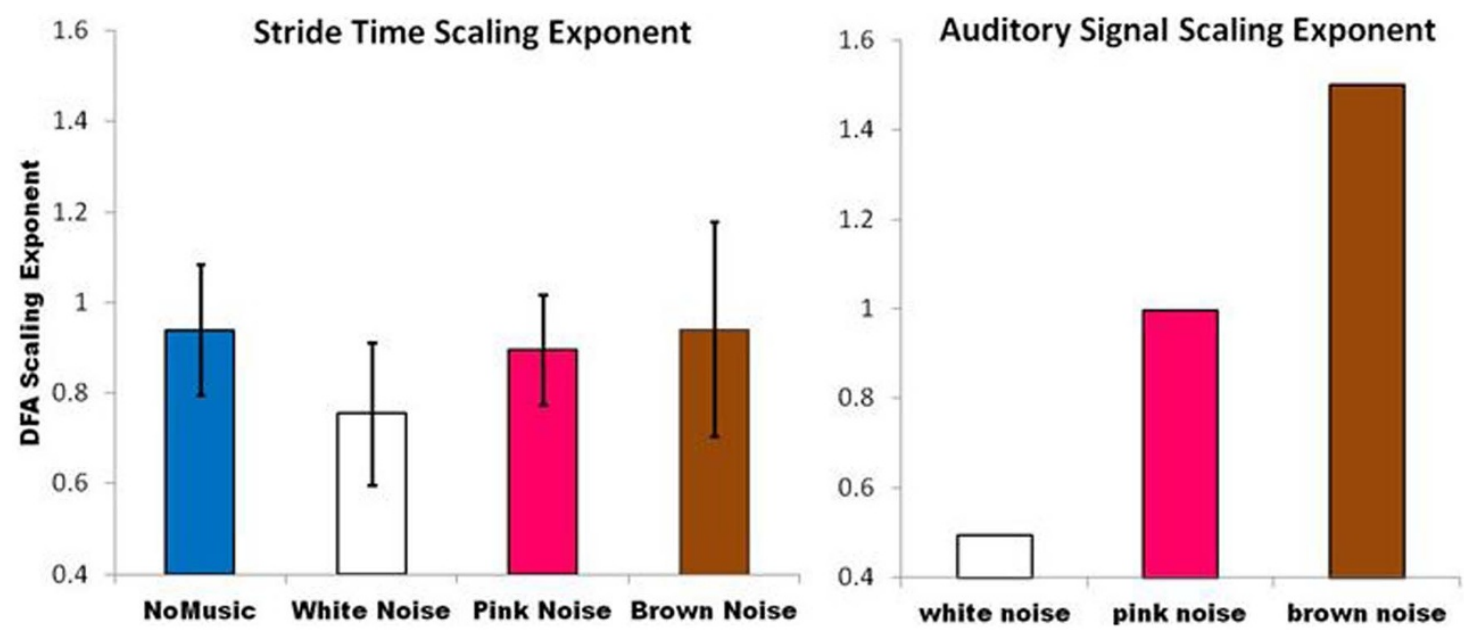

Figure $3 \mid$ Detrended Fluctuation Analysis of the auditory signal is shown on the right for illustration purposes. Detrended Fluctuation Analysis of the stride time series across the four different walking conditions, for all subjects, is shown on the left. Error bars are standard deviations. 
using variable auditory stimuli to drive gait dynamics. This study showed that older adults tended to entrain to the variable auditory stimuli more readily than the younger adults. The robust fractal structure observed across conditions in the present study in young, healthy adults further substantiates this finding. We would therefore be reluctant to generalize the findings of this study to older or infirmed cohorts. We have begun new studies applying the method reported here to older adults, and our preliminary data (see supplementary material 2) suggest that older adults may indeed demonstrate greater modifications to gait structure when listening to fractal auditory stimuli, due in part to an altered baseline level of fractal scaling in the motor time series.

The potential of rhythmic auditory cueing in motor rehabilitation is well recognized. However, complex auditory cues with varying statistical properties represents a novel development in the field. There are many unanswered questions, for example: 1) which type of complex signal is most likely to improve pathological gait in an uncued condition? 2) which type of complex signal is most likely to lead to improved function while performing activities of daily living? West et al..$^{54}$ suggested that that information exchange is maximal when systems share the same complexity, and especially $1 / f$ scaling. Given that dynamics similar to pink noise, i.e. 1/f scaling, is characteristic of healthy human walking, it seems logical that an auditory stimulus for gait rehabilitation should be based on this presumably optimal structure ${ }^{31}$. For robust motor control we need to quickly instigate a successful strategy from an unexpected state. In biological systems, this seems to require continual exploration, even after the point of movement mastery. Maintaining robust control of walking in the event of a perturbation that drives the system from a highly probable state to a less probable one e.g. encountering slopes, steps, stairs, rough terrain, slippery surfaces, negotiating static, moving and unexpected obstacles requires a rich repertoire of movement strategies. It is important that humans continue to invoke a structured variability that enables us to explore and experience the less probable states in order to maintain a robust control strategy. The diverging and folding nature of complex systems produces deterministic variability which may allow us to safely explore a larger region of state space around our walking attractor. The use of an auditory stimulus such as the pink noise music stimulus presented here is likely to drive a particular learning process in which the parameters governing the temporal structure of gait variability are explored until the system self-organizes to produce a matching fractal structure. This is likely to result in more stable yet flexible walking in an uncued condition, developing functional, adaptive capabilities for successful navigation of a real-world, dynamic environment. There is a pressing need for future work to test these hypotheses so that older adults and neurologically impaired patients can benefit from the enormous potential this theoretical framework has to offer the field of rehabilitation science.

While this study and previous studies ${ }^{32,33}$ quantify changes in statistical properties of time series due to external cueing, it is more than a little challenging to attempt to interpret the clinical significance of these changes. The magnitude of the main effects reported here for the repeated measures Anova (i.e. $\omega^{2}=0.12$ for scaling exponent and $\omega^{2}=0.15$ for sample entropy) could arbitrarily be interpreted as medium to large, however there is currently no empirical framework on which to authenticate this interpretation. In order for this field of research to be translated into operational rehabilitation models, randomized control studies are required that enable meaningful and consistent interpretation of changes in entropy and fractal measures induced by interventions such as the one presented here.

Advances in technology should enable the design and deployment of a bi-directional adaptive dynamical system as a rehabilitation tool. Recent studies have shown that an interactive, bi-directional approach strengthens the effect of the entrainment ${ }^{31,50}$. Future studies will seek to close the loop on the signal flow in which footswitches create sounds that play notes along with the music that the person hears, leading to significantly greater entrainment as it includes a participatory element in the rhythmic auditory stimulus as well as a feedback signal (in addition to the proprioceptive feedback already present). This feedback signal combined with the music would act as an error signal to facilitate accelerated learning.

It is accepted that entropy and fractal based approaches of time series analysis are data-hungry techniques, yet the practical challenges of capturing an adequately long gait time series for rigorous investigations of the above questions in elderly or infirmed cohorts are not trivial. Recent studies ${ }^{55,56}$ have highlighted potential problems when using DFA for the quantification of persistence in time series. Bryce et al. ${ }^{55}$ demonstrates that DFA systematically underestimates dispersion and that this bias is sample size dependent. Schaefer et $\mathrm{al}^{56}$ highlighted the inherent limitations of common methods used to quantify scale invariant processes and similarly reported that analysis of time series of $n<100$ using DFA can return inaccurate results. The authors have also previously reported on the perils of utilizing entropy measures for short time series ${ }^{43}$. While the time series captured in this study was of length $n=512$, we are still mindful of the methodological limitations of these widely used techniques and recommend caution for the reader also. Development of more appropriate techniques for the analysis of motor time series is beyond the scope of the current study, but it is predicted that this field will see further advancements in the near future. Equally, the use of complex auditory signals as a rehabilitation tool to restore biological complexity to the locomotor system is an area ripe for progression along the translational research spectrum. The research questions discussed here may serve to guide these advancements.

1. Sejdic, E. et al. The Effects of Rhythmic Sensory Cues on the Temporal Dynamics of Human Gait. PLoS One 7, e43104 (2012).

2. Larsson, M. Self-generated sounds of locomotion and ventilation and the evolution of human rhythmic abilities. Anim Cogn 17, 1-14 (2013).

3. Baram, Y. \& Miller, A. Auditory feedback control for improvement of gait in patients with Multiple Sclerosis. J Neurol Sci 254, 90-94 (2007).

4. Roerdink, M., Bank, P. J. M., Peper, C. E. \& Beek, P. J. Walking to the beat of different drums: Practical implications for the use of acoustic rhythms in gait rehabilitation. Gait \& Posture 33, 690-694 (2010).

5. Rochester, L. et al. Does auditory rhythmical cueing improve gait in people with Parkinson's disease and cognitive impairment? A Feasibility study. Mov Disord 24, 839-845 (2009)

6. Wittwer, J. E., Webster, K. E. \& Hill, K. Rhythmic auditory cueing to improve walking in patients with neurological conditions other than Parkinson's disease: what is the evidence? Disabil Rehabil 35, 164-176 (2013).

7. Thaut, M. H. et al. Rhythmic Auditory Stimulation Improves Gait More Than NDT/Bobath Training in Near-Ambulatory Patients Early Poststroke: A SingleBlind, Randomized Trial. Neurorehabil Neural Repair 21, 455-459 (2007).

8. Schmidt, R. A schema theory of discrete motor skill learning. Psychol Rev $\mathbf{8 2}$, 225-260 (1975).

9. Stergiou, N., Harbourne, R. T. \& Cavanaugh, J. T. Optimal movement variability: a new theoretical perspective for neurologic physical therapy. JNeurol Phys Ther 30, 120-129 (2006).

10. Costa, M., Goldberger, A. L. \& Peng, C. K. Multiscale Entropy Analysis of Complex Physiologic Time Series. Phys Rev Lett 89, 068102 (2002).

11. Peng, C. K. et al. Quantifying Fractal Dynamics of Human Respiration: Age and Gender Effects. Ann Biomed Eng 30, 683-692 (2002).

12. Buzzi, U. H. et al. Nonlinear dynamics indicates aging affects variability during gait. Clin Biomech 18, 435-443 (2003).

13. Goldberger, A. L., Rigney, D. R. \& West, B. J. Chaos and fractals in human physiology. Sci Am February 262, 42-49 (1990).

14. Goldberger, A. L. Heartbeats, hormones, and health: is variability the spice of life? Am J Respir Crit Care Med 163, 1289-1290 (2001).

15. Goldberger, A. L. et al. Fractal dynamics in physiology: Alterations with disease and aging. Proceedings of the National Academy of Sciences of the United States of America 99, 2466-2472 (2002).

16. Goldberger, A. L., Peng, C. K. \& Lipsitz, L. A. What is physiologic complexity and how does it change with aging and disease? Neurobiol Aging 23, 23-26 (2002).

17. Lipsitz, L. \& Goldberger, A. Loss of 'complexity' and aging. Potential applications of fractals and chaos theory to senescence. JAMA Apr 267, 1806-1809 (1992).

18. Lipsitz, L. A. Dynamics of stability: the physiologic basis of functional health and frailty. J Gerontol A Biol Sci Med Sci Mar 57, 115-125 (2002).

19. Manor, B. et al. Physiological complexity and system adaptability: evidence from postural control dynamics of older adults. J Appl Physiol 109, 1786-1791 (2010). 
20. Dossey, L. Coherence, Chaos, and the Coincidentia Oppositorum. Explore (New York, NY) 6, 339-345 (2010).

21. Coffey, D. Self-organization, complexity and chaos: the new biology for medicine. Nat Med August, 4, 882-885 (1998).

22. Kiss, R. M. Effect of severity of knee osteoarthritis on the variability of gait parameters. J Electromyogr Kinesiol 21, 695-703 (2011).

23. Hausdorff, J. M. et al. Altered fractal dynamics of gait: reduced stride-interval correlations with aging and Huntington's disease. J App Physiol 82, 262-269 (1997).

24. Hausdorff, J. M. et al. Gait variability and basal ganglia disorders: stride-to-stride variations of gait cycle timing in Parkinson's disease and Huntington's disease. Mov Disord 13, 428-437 (1998).

25. Herman, T., Giladi, N., Gurevich, T. \& Hausdorff, J. M. Gait instability and fractal dynamics of older adults with a "cautious" gait: why do certain older adults walk fearfully? Gait \& Posture 21, 178-185 (2005).

26. Hausdorff, J. M. Gait dynamics, fractals and falls: Finding meaning in the strideto-stride fluctuations of human walking. Hum Mov Sci 26, 555-589 (2007).

27. Paterson, K., Hill, K. \& Lythgo, N. Stride dynamics, gait variability and prospective falls risk in active community dwelling older women. Gait \& Posture 33, 251-255 (2009).

28. Toebes, M. J. P. et al. Local dynamic stability and variability of gait are associated with fall history in elderly subjects. Gait \& Posture 36, 527-531 (2012).

29. Montero-Odasso, M., Muir, S. W. \& Speechley, M. Dual-Task Complexity Affects Gait in People With Mild Cognitive Impairment: The Interplay Between Gait Variability, Dual Tasking, and Risk of Falls. Arch Phys Med Rehabil 93, 293-299 (2012)

30. Wu, H. et al. Temporal structure of motor variability is dynamically regulated and predicts motor learning ability. Nat Neurosci 17, 312-321 (2014).

31. Hove, M. J. et al. Interactive Rhythmic Auditory Stimulation Reinstates Natural 1/ f Timing in Gait of Parkinson's Patients. PLoS One 7, e32600 (2012).

32. Marmelat, V., Torre, K., Beek, P. J. \& Daffertshofer, A. Persistent Fluctuations in Stride Intervals under Fractal Auditory Stimulation. PLoS One 9, e91949 (2014).

33. Kaipust, J., McGrath, D., Mukherjee, M. \& Stergiou, N. Gait Variability is Altered in Older Adults When Listening to Auditory Stimuli with Differing Temporal Structures. Ann Biomed Eng 41, 1595-1603 (2013).

34. Hausdorff, J. M. et al. Fractal dynamics of human gait: stability of long-range correlations in stride interval fluctuation. J App Physiol 80, 1448-1457 (1996).

35. Delignieres, D. \& Torre, K. Fractal dynamics of human gait: a reassessment of the 1996 data of Hausdorff et al. J App Physiol 106, 1272-1279 (2009).

36. Terrier, P. \& Dériaz, O. Persistent and anti-persistent pattern in stride-to-stride variability of treadmill walking: influence of rhythmic auditory cueing. Hum Mov Sci, (2012).

37. Terrier, P., Turner, V. \& Schutz, Y. GPS analysis of human locomotion: Further evidence for long-range correlations in stride-to-stride fluctuations of gait parameters. Hum Mov Sci 24, 97-115 (2005)

38. Repp, B. \& Su, Y.-H. Sensorimotor synchronization: A review of recent research (2006-2012). Psychon Bull Rev 20, 403-452 (2013).

39. Lindsay, T. R., Noakes, T. D. \& McGregor, S. J. Effect of treadmill versus overground running on the structure of variability of stride timing. Percept Mot Skills Apr, 118, 331-346 (2014).

40. Dingwell, J. B. \& Marin, L. C. Kinematic variability and local dynamic stability of upper body motions when walking at different speeds. J Biomech 39, 444-452 (2006)

41. Keller, P. E., Dalla Bella, S. \& Koch, I. Auditory imagery shapes movement timing and kinematics: Evidence from a musical task. J Exp Psychol Hum Percept 36, 508-513 (2010).

42. Delignieres, D. et al. Fractal analyses for 'short' time series: A re-assessment of classical methods. J Math Psychol 50, 525-544 (2006).
43. Yentes, J. et al. The Appropriate Use of Approximate Entropy and Sample Entropy with Short Data Sets. Ann Biomed Eng 41, 349-365 (2013).

44. Amoud, H. et al. Fractal time series analysis of postural stability in elderly and control subjects. J NeuroEng Rehabil 4, 12 (2007).

45. Havlin, S. et al. Fractals in biology and medicine. Chaos, Solitons, and Fractals 6 , 171-201 (1995)

46. Moraiti, C. O. et al. The Effect of Anterior Cruciate Ligament Reconstruction on Stride-to-Stride Variability. Arthroscopy 25, 742-749 (2009).

47. Smith, B. A., Stergiou, N. \& Ulrich, B. D. Patterns of Gait Variability Across the Lifespan in Persons With and Without Down Syndrome. J Neurologic Phys Ther 35, 170-177 (2011)

48. Cohen, J. Statistical Power Analysis for the Behavioral Sciences 2nd edn. Lawrence Erlbaum. (1988).

49. Stephen, D. G., Stepp, N., Dixon, J. A. \& Turvey, M. T. Strong anticipation: Sensitivity to long-range correlations in synchronization behavior. Physica A 387, 5271-5278 (2008).

50. Marmelat, V. \& Deligneres, D. Strong anticipation: complexity matching in interpersonal coordination. Exp Brain Res 222, 137-148 (2012).

51. Stephen, D. G. \& Dixon, J. A. Strong anticipation: Multifractal cascade dynamics modulate scaling in synchronization behaviors. Chaos, Solitons \& Fractals 44, 160-168 (2011)

52. Stepp, N. \& Turvey, M. T. On strong anticipation. Cogn Syst Res 11, 148-164 (2010).

53. Baer, L. et al. The role of musical training in emergent and event-based timing. Front Hum Neurosci 7, (2013).

54. West, B. J., Geneston, E. L. \& Grigolini, P. Maximizing information exchange between complex networks. Phys Rep 468, 1-99 (2008).

55. Bryce, R. M. \& Sprague, K. B. Revisiting detrended fluctuation analysis. Sci Rep 2, (2012).

56. Schaefer, A., Brach, J. S., Perera, S. \& Sejdic, E. A comparative analysis of spectral exponent estimation techniques for $1 / \hat{f}^{2}$ processes with applications to the analysis of stride interval time series. J Neurosci Meth 222, 118-130 (2014)

\section{Author contributions}

N.H. and N.S. designed the project; N.H. developed the auditory stimuli, performed the experiments and analysed the data with advice from N.S.; N.H., N.S. and D.McG. interpreted the results; D.McG. wrote the first draft of the manuscript; all authors read and approved the final manuscript.

\section{Additional information}

Supplementary information accompanies this paper at http://www.nature.com/ scientificreports

Competing financial interests: The authors declare no competing financial interests.

How to cite this article: Hunt, N., McGrath, D. \& Stergiou, N. The influence of auditory-motor coupling on fractal dynamics in human gait. Sci. Rep. 4, 5879; DOI:10.1038/ srep05879 (2014).

This work is licensed under a Creative Commons Attribution-NonCommercialNoDerivs 4.0 International License. The images or other third party material in this article are included in the article's Creative Commons license, unless indicated otherwise in the credit line; if the material is not included under the Creative Commons license, users will need to obtain permission from the license holder in order to reproduce the material. To view a copy of this license, visit http:// creativecommons.org/licenses/by-nc-nd/4.0/ 